This is an author-created version.

The final publication is available at link.springer.com.

http://link.springer.com/chapter/10.1007\%2F978-3-319-02054-9_43 


\title{
Systematic Procedure for Handling Complexity in the Automotive Production
}

\author{
Florian Schoettl ${ }^{1}$, Nils Herrmann ${ }^{2}$, Maik Maurer ${ }^{1}$, Udo Lindemann ${ }^{1}$ \\ ${ }^{1}$ Institute of Product Development - Technische Universität München, Boltzmannstraße 15, 85748 Garching, Germany, \\ Tel.: +49-89-289-15151, E-Mail: schoettl@pe.mw.tum.de, maurer@pe.mw.tum.de, lindemann@pe.mw.tum.de \\ ${ }^{2}$ Laboratory for Machine Tools and Production Engineering - RWTH Aachen University, Steinbachstraße 19, 52056 \\ Aachen, Germany, Tel.: +49-241-80-27555, E-Mail: nils.herrmann@rwth-aachen.de
}

\begin{abstract}
Volatile influences from global markets force manufacturing companies to be more flexible, innovative and efficient. Implementing these attributes in products, processes and production resources, requires a high rate of change in development periods of decreasing lengths. The increasing level of perceived complexity is a critical result of these changes. Manifold approaches were developed and studies were conducted to derive measures for complex issues. But in the industrial field, the usability of such measures poses a major challenge. In this paper, we bridge the gap between the scientific and the practical perspective on complexity and extract three basic complexity cases. On this basis, our procedure supports the systematic analysis, classification and quantification of complex issues in automotive production. The approach has been successfully applied in an industrial use case of an automotive assembly line.
\end{abstract}

Keywords:

Complexity, Quantification, Automotive Production

\section{INTRODUCTION}

Over the last decades, the manufacturing industry has been facing an increasing number of global changes and fluctuating markets. The most important challenges are variable sales, short development cycles and the necessity to offer individualised and ecological products. In order to ensure economic success in the future, every business unit has to meet these new requirements. The production and it's periphery tend to show the most problems with meeting those requirements as they are dependent on the results of the technical development, planning and operation with all of its possible faults. Hence, effects on the production should be focused on when making strategic decisions about enlarging the product portfolio, using innovative production technologies, constructing new parts or building new plants.

In the automotive industry, the understanding of current changes in the company's environment and the knowledge about their internal impact play a key role for strategic decisions. These external changes build a dynamic net of requirements, boundary conditions and target values with an increasing level of complexity that is reflected in production sites. This trend was already postulated by Asbhy's law of requisite variety in the middle of the 20th century, based on considerations of system theory [1]. Therefore, volatile external factors cause a need for changeable processes and flexible production resources - an agile production system. There is a strong interaction between the complexity of a production system and its agility, so that more changeability of resources means increasing complexity because of more degrees of freedom and possible dynamics.

In the industrial field, there is still a lack of awareness for the multiple impact of complexity in the production, because no established description models or methods for a systematic handling are available. Therefore, measures are taken, that usually solve the occurring problems for a short period of time. Our goal is to increase transparency in dealing with complexity and to support the analysis, assessment and finally the selection of appropriate measures by a procedure model. Our contribution introduces the definition of three prevalent complexity cases and a quantification model for complexity, which is embedded in a procedure model.

In section 2, we briefly discuss the current research regarding the nature of complexity, characterise the perception of complexity in the industrial field and give an overview of present strategies. In section 3 we define three cases to classify practical complexity problems. Section 4 describes the six single steps of our approach in detail. A case study of an automotive assembly line shows the practical application of our method in section 5 .

\section{THE NATURE OF COMPLEXITY}

The difficulty of making complexity describable and assessable is the motivation for our approach, as shown in Figure 1. This problem originates from the nature of complexity and becomes apparent while looking at the main issues from the scientific and the practical point of view. Hence, we discuss complexity from both points of view, work out the current challenges in handling complexity and point out our contribution in the following subsections.

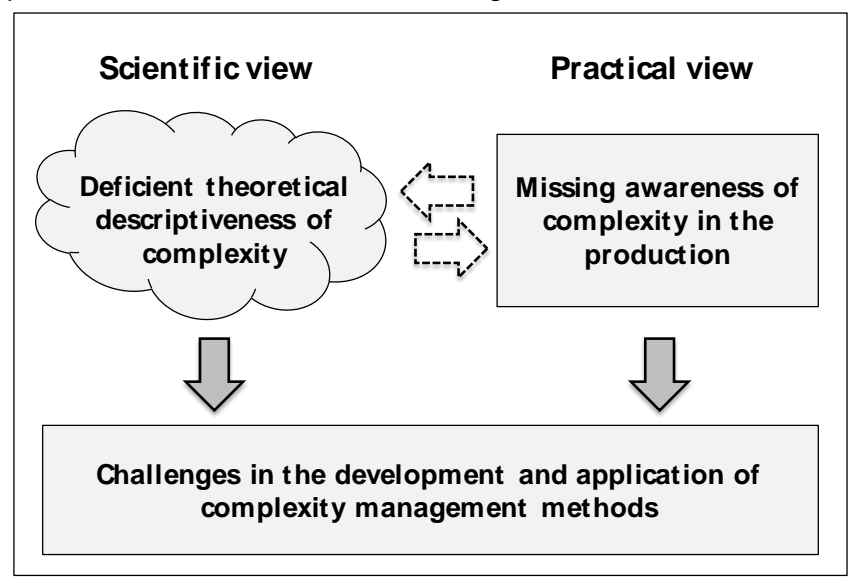

Figure 1: Motivation for a systematic procedure for handling complexity. 


\subsection{Complexity as system state}

Today, the term "complex" is used more and more inflationary. The discussion about complexity increases in the same way as society, economy and environmental phenomenon changes or rather the awareness of their dependencies raises. But there is no clear definition of what exactly is meant by calling something complex. Nicolis and Prigogine characterise complexity as a term whose definition is an essential part of the problem complexity poses [2]. In literature, many different sciences are concerned with the explanation of complexity and consequently, a lot of descriptions and definitions can be found [3]. The fact all considerations have in common is paraphrased by Flood: "In general, we associate complexity with anything we find difficult to understand" [4]. This statement contains two important aspects, which help to understand complexity. There is always a person "we", who appreciates the difficulty and an object "anything" that has specific properties. Hence, complexity occurs only during the interaction of a subject (person) with an object, but not as property or state of an item or circumstance. For example, running a machine or planning the layout of a shop floor may be complex tasks, but neither the machine nor the layout plan are complex objects.

The description of complexity can be enlarged by a system theory perspective, if the person interacting with the object and the object itself are understood as a system. According to Cotsaftis, simple, complicated and complex are states a system can be characterise with [5]. Maurer combines these system states with structural system attributes (the number of dependencies, the number of system elements and the existence of dynamic), which allows a clear differentiation and subsequently precise classification of systems. In addition, Maurer takes the user into account and describes the interaction with the system [6]. Schuh et al. transfer the mentioned system states explicitly on production systems and distinguish four occurring types of systems: simple, complicated, complex, complicated and complex. Hence, they divide up production systems into a complicated and complex part [7]. Coming from the same three states, this comparison clearly shows that complexity is related significantly different to the system. It becomes obvious that different conclusions are drawn and that there is no common classification of complexity even from an abstract point of view.

\subsection{Complexity in the industrial field}

Modern production systems consist of a high number of strongly connected elements with internal and external dynamics. Different production strategies, products, processes, machines and boundary conditions build a broad area of possible complexity cases. Because of the system size, which grows with each change, it becomes more and more difficult to keep track of all causal connections. In the past, a planner could describe the activities of a work station, for example the mounting of a car's frontend, only by the knowledge of the input and output. Nowadays, it is necessary to take also not directly previous assembly activities into account, like the installation of a radar sensor which has to fit with the frontend. The current trend poses increasing challenges in planning and operating production systems, but is complexity the right explanation of this phenomenon or even the reason?

Even though there are a concrete field of observation and describable problems, no resilient definition of complexity can be given from the industrial point of view. So, it's not surprising that everybody quotes to have a complex job or has to cope with complex problems. The people working in the automotive industry, we have interviewed in our case study confirmed that impression. There are some feasible reasons for it. First, having a complex job can be interpreted as "distinction" and emphasises the importance of someone. This has its seeds in missing objective agreements concerning complexity, because no obligatory assessment and quantification methods are established. A handicap for introducing such methods is the demand for making complexity measurable, exclusively by costs. Based on scale effects, some approaches were developed in terms of complexity costs induced by variety (cp. [8], [9]). But reliable approaches, which consider all relevant influences, are not known. Another aspect is the often missing institutionalisation of complexity management in producing industry or even the missing person in each project team which is responsible for the consideration of complex issues. The insufficient implementation in the organisation seems to be similar to former changes in terms of quality management.

\subsection{Challenges in handling complexity}

As shown in the previous explanation of the theoretical and the practical view on complexity, a consistent and universally applicable form of description is missing. So, it is obvious that the handling of complexity results in a similar range of scientific approaches and practical case studies. In both fields, one can find the keywords avoid, reduce and control. Lindemann et al. quote the three measures in terms of Structural Complexity Management based on the objects market, product, process and organisational complexity [10]. But selecting the best fitting measure depends on the necessity of the occurring complexity. This demands a previous assessment.

From a system theory perspective, complexity can be defined by characteristics of basic system attributes (cp. [6]). In literature, there are only a few approaches that are generally applicable to assess and measure the system complexity using these attributes. Most of them focus either on products or on manufacturing systems [11]. But based on these three facts, a useful and purposeful assessment is not possible, because the impact of complexity in a technical system depends on the interaction with the system user, e.g. a production planner or machine operator. Heylighen sees the key problem in the decomposability of a complex system, because it handicaps the modelling, the usage of models, the interpretation of results and finally the development of a simple assessment method [12]. The approach of Feliz-Teixeira and Carvalho Brito also depicts the difficulty arising from a universal assessment without a concrete reference object [13]. Their proposed model focuses the holistic interpretation of system behaviour by the use of system simulation combined with Fourier-analysis and quantum mechanics. This discrete approach needs to be evaluated in a real use case.

Marti's Complexity Management Model is a more realistic approach for the assessment of product complexity [14]. He differentiates between internal and external complexity, whose balance poses the objective of this method. Internal complexity results from the multitude and variety of elements (components) and correlations to products, similar to the previously mentioned system attributes. Coming from a complexity induced change of the product architecture, he derives recommended activities in a three step procedure. Practical approaches aim almost exclusively at the early avoidance or the belated reduction of product variety. This kind of complexity management is synonymous to the well-known variant management, because only negative and direct influences of variety are observed. In the case of influenced assembly processes, Samy and EIMaraghy present a detailed approach based on dependency matrices [15]. Regarding current trends in automotive industry, the increasing number of variants is not per se bad, but rather essential to be able to offer a growing and attractive product portfolio.

Consequently, it becomes clear that in practical action there is hardly any causal context between the occurring problem, the presence of complexity and appropriate measures. Numerous examples from companies, where product variants were removed 
from the portfolio due to a supposed reduction of costs and complexity serve as proof. The frequent use of additional resources in terms of employees and an increasing invest for solving problems, demonstrates the necessity for a strategic handling of complexity by a systematic approach. The literature review about analysis and assessment methods indicates that no approach exists, which copes with the disclosed problem.

\section{AUTOMOTIVE PRODUCTION - A COMPLEX SYSTEM FROM THE USER'S PERSPECTIVE}

Starting from the problems on the scientific and the practical side identified in the previous section, we have developed a basic understanding of complexity, which is shown in three specific complexity cases. Based on these definitions, the question is whether a system designed, engineered and built by humans can ever be called a complex product. We argue that complexity is a subjective perception of the person who interacts with a complex system. In relation to the issues that arise in production systems, this view is consistent with the approaches of Landry et al. and Fox, that problems cannot be understood as objective realities that exist independently of the subjects [16], [17]. Even if the problem objectively exists, a subjective view of the parties is always reflected in the problem solving process. This subjectivity depends on factors like the background knowledge of the person.

The transfer of this definition into existing processes in productive systems brings up three major cases in which those attributes can be true, visualised in figure 2 .

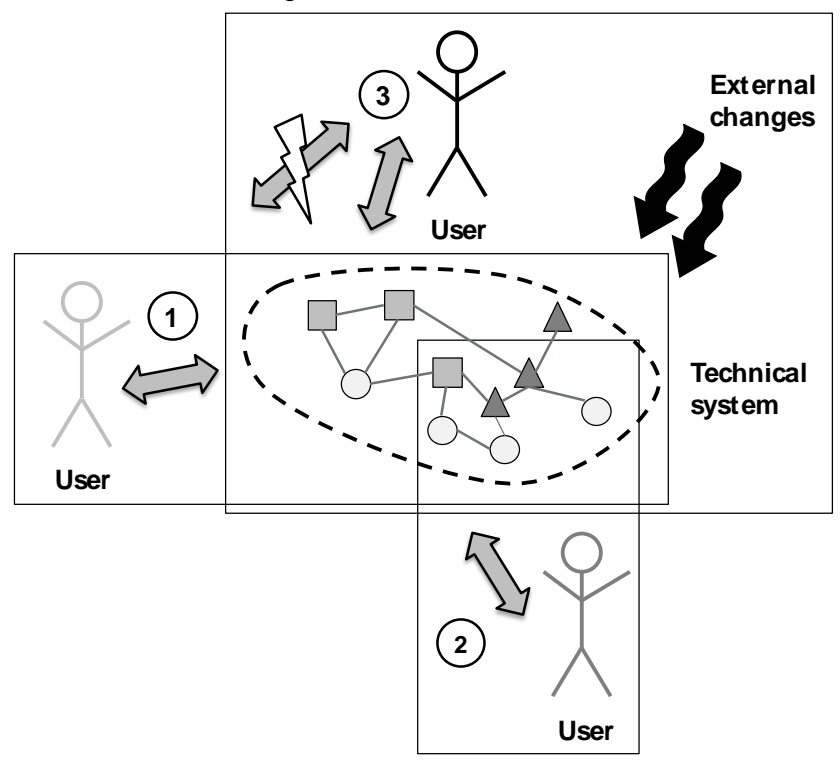

Figure 2: Complexity cases.

\subsection{Lack of information under dynamic influence (1)}

It is conceivable that the working environment of a single person or a group changes relatively to the current known state. The perceived dynamic arises from temporary effects in the system environment, which influence the environment. The user does not know about the source of this dynamic influence and its impact. Despite of this lack of information, the system is describable at a particular time, so that all system elements are known and dependencies become clear. After a finite length of time, there is no longer a lack of information and the system is perceived as complicated instead of complex.

The mentioned scenario can be found in the planning of production systems. During the planning activities, dynamic effects which cause complexity cannot be considered, because they are not known. Otherwise they would be compensated. Consequently, there is a static system design in the end of the planning process which behaves static and robust in the real implementation. But it is immediately evident that such an optimal case can only appear under particular circumstances in reality. The climate in a factory work floor can strongly influence the production process, for example the temperature and humidity which can interfere with welding and bonding processes. Based on such incidents, a static system can easily become dynamic if the incident is significant for the system stability. Due to this, the behaviour of our completely described production system is quickly perceived as complex by the user. A certain rest of complexity remains, which is caused by effects outside the user's scope. In this case, elimination measures for dynamic system changes characteristically take effect in the beginning, but not for the next time. Hence, the information about external influences concerning the occurring problem was insufficient.

\subsection{Lack of information under quasi-static influence (2)}

In the previous subsection described systems with dynamic influences can be perceived in a snapshot as static, non-complex systems. Such a system is in a stable state when it has settled and all the dynamic effects were compensated for example by control. Nevertheless, the system can be considered as complex when the cognitive performance of the user is not sufficient for the system size and the density of the inherent relations.

Referring that facts to the working environment of the user, this situation means that he loses track of the system either by a current training process (solvable complexity problem) or by the number of dependencies in the system (not solvable complexity problem) Thus, the system seems to be complex and dynamic although it actually remains in a static state. In addition to the density of relations, the number of tasks can also prevent the user from being aware of all links and taking them into account according to their causality for problem solving. Finally, the user cannot decompose or systemise the problem. This situation especially occurs in change processes in the production. In this paper a system like that is called quasi-static, as it only seems to be non-transparent and consequently complex for the user.

Finally, one could state that any system can be transformed into a quasi-static condition. This requires that the field of observation is chosen large enough and all influencing factors are analysed and described according to their potential impact. But this is unrealistic compared to the expense and the potential speed of analysis and therefore, dynamic systems have a right to exist.

\subsection{Interrupted information flow (3)}

In addition to the consequences of the size and the inherent degree of crosslinking of the system and the cognitive abilities of the user, there is another aspect that can lead to a lack of information to the user.

In large systems, information is often shared via a multitude of different points of interaction between the actors in the system. Here the single planner $A$ is not aware that planner $B$ requires information to solve his problem. At the same time, planner $B$ does not know that planner $A$ has the necessary information and thus did not ask him. If the number of interfaces which are needed by the user to interact with the system increases, the probability that he consciously or unconsciously does not have all information also rises. Thus, a non-communicated system change in one area can negatively influence the perceived complexity in a other area, although a comprehensive system overview is provided. 


\section{SYSTEMATIC HANDLING OF COMPLEXITY}

Based on the developed definitions of complexity, as a practical interpretation of the theoretical understanding, the same approach leads to an application-oriented model that supports the management of complexity. Our approach of handling complexity includes six standard steps and enables an objective assessment of a subjective perception of the system in a systematic and sustainable way. Starting with the problem description, the single steps are sequentially passed through up to the selection of measures. In certain situations, it is possible to skip a step. The premises for those situations are explained in detail in the following subsections.

Figure 3 visualises the procedure, which begins by analysing the occurring complex issue. Afterwards, the problem is clearly classified and differentiated into complicated issues by using the three different cases of occurring complexity mentioned in section 3. To identify the causal parameters, all sources of the considered problem and the related departments have to be determined. After assessing the complex issue in terms of valuable and valueless complexity, the quantification follows in a separate model, which is based on the identified parameters. In the end, appropriate measures can be selected and used to handle the system complexity. Because of the subjective perception of complexity, as already pointed out in section 3 , we advise to execute step 2 and step 5 in a larger group of planners to minimize individual influences.

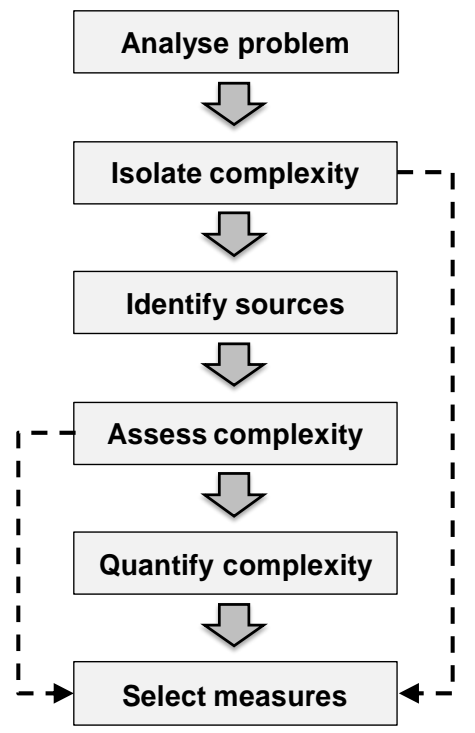

Figure 3: Procedure model for handling complexity.

\subsection{Analyse problem}

The problem analysis (respectively problem description) is the firs step of the procedure and aims at the characterisation of the supposed complex situation in order to subsequently isolate the complexity. For that purpose, we have formulated key questions in order to be able to classify the situation by local, temporal and modal aspects. Since the problem appears with a negative impact, first the negative phenomena which occur in this context are described. Then, the relevant objects (products, processes, resources or a combination of them) which are linked to the problem are identified. Consequently, the temporal aspects are clarified, when and how often the problem occurs, which indicators were considered and how long the problem existed until it was discovered. As a last step, it is important to identify at which position in the process the problem occurred, who has seen it, and in whose area of responsibility it falls, so that the identification of the source is possible.

\subsection{Isolate complexity}

After the problem is comprehensively described, a characterisation on the basis of these criteria divides it into complex or just complicated situations. It is also decided, if further specification of the problem is needed. This characterisation allows a classification in the complexity cases mentioned in section 3 . There is no strict guideline, but the criteria are indications and may be assigned as follows:

- Location of occurrence and responsibility: Dimension of the system boundary and position of the user.

- Temporal aspects: Localisation of the lack of information and existence of external influences.

- Modal aspects: Affected system elements and criticality of the problem.

After this characterisation, if no link is made to a complexity case, it is most likely just a complicated situation. To confirm this hypothesis, some more general questions that are based on the basic properties of complex systems can be answered, e.g.:

- Is the problem reducible or decomposable? Would it be easier to solve the problem with several people?

- Is it a problem whose consequences can be deterministically described and can it consequently be solved by effort?

If these questions can be answered with yes, one can jump to the selection of measures, which are appropriate for complicated problems.

\subsection{Identify sources}

If the presence of a complex issue was found in the previous step, the sources are identified now. In this context, a source means a parameter which is defined and changed outside the field of observation. The goal is to identify all the relevant complexity drivers for the problem and determine who is originally responsible for them. It is necessary to distinguish whether the responsibility lies with a neighbouring department of planning or operation, which works on the same hierarchy level or with an area outside the production system (e.g. marketing). Moreover, the interaction of sources has to be examined, because this information is essential for the subsequent assessment and quantification.

\subsection{Assess complexity}

After going through the steps 1 to 3 , the problem is described in sufficient detail in order to be able to start the analysis. Basis for the next steps and finally the application of appropriate measures is to assess the occurring complexity in terms of valuable and worthless. The value-adding processes in production are relevant for the assessment. If the complexity is related to non-value-adding processes, such as travel paths of employees, it should be at least reduced or if possible completely avoided. The same applies for supposed value-adding processes, such as producing an additional component version which finally contributes no commercial value because it addresses no additional customers.

If it is complexity, which occurs in the context of value-adding processes and also contributes to the economic success, we talk about valuable complexity. This may be for example an additional engine variant a market niche is occupied by or by which customers will be won from competitors. In this case, the associated complexity must be handled as effectively as possible. Then, we speak about the control of complexity. 


\subsection{Quantify complexity}

If the previous assessment has shown that the examined problem is associated with worthless complexity, one can directly jump to the selection of appropriate measures. Otherwise, the quantification has to follow in order to be able to describe the criticality of the problem by the relative complexity increase or decrease.

As defined earlier, complexity rises in the user's perception within the interaction process with the system. Complexity is no explicit system variable, its quantification depends on the user's background experience and on the patterns he uses to evaluate situations. To quantify the perceived complexity, it is necessary to have a unified language and a consistent structure. Therefore, we have developed a structure for the evaluation process that is, because of its degree of abstraction, transferable between the subsystems of a production system. This makes its results comparable to earlier evaluations. The practical use depends on the quickness of conduction and simplicity of the evaluation process. The process is shortened by knowledge transfers between evaluations in different subsystems and over time between evaluations in the same. Figure 4 visualises the quantification model consisting of seven steps. How to pass through the single steps depends on the particular application.

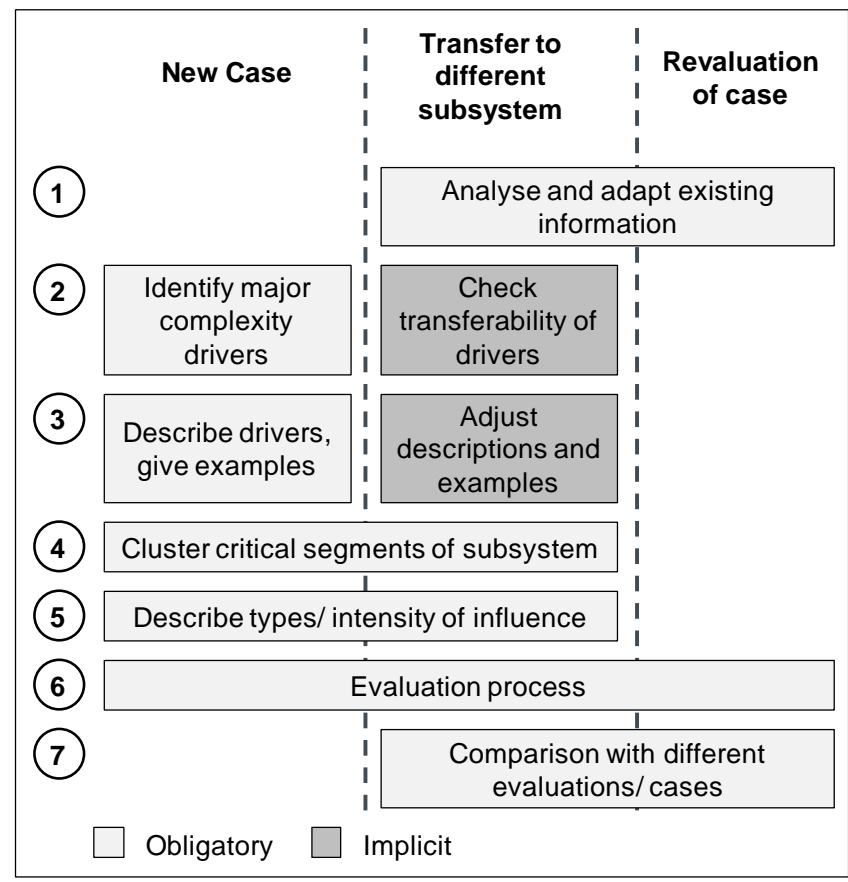

Figure 4: Quantification procedure

The model differentiates if the user is confronted with a new problem (2 to 6 ) which has never been evaluated before, if he wants to transfer the evaluation process to a different element of the production system ( 1 to 7 ) or if there is already data of an earlier evaluation which shortens the expansion for the evaluation $(1,6,7)$. The boxes coloured in dark grey depict implicit steps, which are already included in the first step.

The first step of the procedure is the analysis of existing information if there were earlier evaluations. It has to be checked if the evaluation strategy of an earlier case fits with the current problem. In a second step, the major complexity drivers have to be aggregated to a small, abstract and well-defined collection. The third step is the description about what is behind those abstract drivers concerning the specific subsystem. The fourth step is about the clustering of the subsystem in single parts, which can be evaluated individually. They must be sufficiently separated from each other. In the fifth step, the impact of a complexity driven problem on other system parts has to be described and assigned to the specific criticality classes. Therefore, we differentiate between significant criticality, normal criticality, less criticality, and no system influence. For the evaluation in the sixth step the assessor has to describe under which conditions he would rate a problem accordingly. The sixth step is about the assignment of complexity driven problems to the system influence criticality by reference to the specifications generated in step five. Summing up the specific numeric values assigned to the criticality classes can generate a global value of the system complexity. If there is already a numerical value scale, it has to be adapted to guarantee comparability. If not, we suggest using the 6-3-1-0 emphasis with decreasing criticality, which is also used in our case study. If there was an earlier evaluation, it is possible to compare the results in a seventh step.

\subsection{Select measures}

In the selection of measures, we have to distinguish between valuable or worthless complexity which arises with the initial problem. In the first case of valuable complexity, one of the most important steps have already been done by the quantification, because this procedure already contributes significantly to increased system transparency. In terms of our three complexity cases, this means reducing the lack of information. In addition, different forms of visualisation can be used to further increase the transparency of the system and control significant complexity drivers by redesigning the product architecture or the transformation of the production architecture (production processes, resources and its dependencies).

In the case of worthless complexity, the causal processes, products or system states that occur should be avoided. This can be done by well-known tools of lean-thinking, e.g. ABC-analysis. If a fully avoidance is not possible, one has to reduce the occurrence of complex situations by defining barriers for certain parameters and eliminate problematic combinations. The operation of a manufacturing system in a better controlled parameter range where negative external influences are reduced to a minimum would be a practical example.

\section{CASE STUDY: COMPARISON OF TWO AUTOMOTIVE ASSEMBLY PROJECT STATES CONCERNING ITS INHERENT COMPLEXITY}

For the validation of the theses referring to complexity and its characteristic definitions in relation to its occurrence in automotive production, the assembly process of a frontend was examined after a model change. It states as example for the analysis of a whole automotive assembly line because the identified assessment aspects are valid in all areas of this system.

When assembling the frontend to the car, problems with the installation of the radar sensor were occasionally detected. This failure was first discovered by putting the car in operation and testing the driving assistance systems. Both the assembling and the testing employee did not know any reasons for the problem. By using the earlier described complexity cases, it was ensured, that the analysed problems arose from complexity case number 2 . The installation of the radar sensor serves as a proper example for this case, because its assembly requires special knowledge and experience. Furthermore, it has a lot of dependencies to the surrounding parts, following assembly processes and finally to the operability of the whole car. In the next step, sources and complexity enhancing aspects had to be identified. Therefore, the production planning and the operating divisions were interviewed to 
allocate the responsible department. It became clear that the planning department did not know about the special difficulties in the assembly process of this sensor and kept the established process without informing the assembly employees about the design change of the sensor. The assessment of the problem revealed that the occurring complexity is caused by a changed sensor fixation due to a newly designed frontend. This increases the attractiveness for customers and is therefore a case of valueadding complexity.

To quantify the relative increase of complexity and the consequent criticality of the problem, the relevant assembly segment was isolated and examined for the effect of complexity causes. After that, the resulting complexity drivers were accumulated in more abstract groups:

1. Special variation of assembly / Variance in the assembly process

2. Workload, time pressure, stress

3. Variance in tasks

4. Personal competence

5. Ergonomic circumstances

6. Quality standard

7. Usage of utilities, supportive systems

These key aspects were rated regarding their impact on the system. The impact was significantly critical because the mistake in the assembly of the radar sensor influences downstream processes and was discovered late in the process during the final function test. In assessing the relative complexity increase, compared to the situation before the model change, it showed that an increase from 17 to 64 was found. The higher numerical value is consistent with the perceived increase of complexity. Since it is a valuable complexity, visualising the link between the new setting of the radar sensor and the modified installation parameters could be a useful measure.

\section{CONCLUSION AND OUTLOOK}

This contribution introduces a method for analysing, assessing and quantifying the system complexity in a six step procedure considering specific requirements of usability and target values of an automotive production system. Based on the defined complexity cases from a practical view, focused on the user, complicated and complex problems can be distinguished and appropriate measures can be selected. Since the problem and its causes are systematically identified, measures can be taken purposefully. Through the evaluation of the critical aspects it is possible to find the most critical areas in the system. The cumulated values make it easy to compare the aspects and to identify where a high priority of measures is. The quantification of the relative increase or decrease of complexity allows the comparison of several system states and provides an objective discussion of complexity impact on the production system. Thus, the early dialogue between departments involved in the production process is supported, which results in lower long-term lack of information and consequently in a better control of the perceived complexity. However, further applications to secure the method and to allow a more accurate interpretation of this complexity value are needed. The goal is to define a critical value and derive the maximum tolerated complexity for the production system. For this purpose, the approach will be expanded by the influence of structural criteria in the future. A link to assessment methods for the adaptability of production systems is also planned.

\section{REFERENCES}

[1] Ashby, W. R. (1958): Requisite variety and its implications for the control of complex systems, in: Cybernetica, Revue trimestrielle de l'Association Internationale de Cybernétique, Vol.1, No. 2, pp. 83-89, Assoc., Namur.

[2] Nicolis, G.; Prigogine, I. (1989): Exploring complexity: An introduction, W. H. Freeman, New York.

[3] Talley, D. N. (2008): Methodology for the conceptual design of a robust opportunistic system-of-systems, Diss., Georgia Institute of Technology.

[4] Flood, R. (1993): Dealing with Complexity: an introduction and application of systems science, Plenum Press, New York.

[5] Cotsaftis, M. (2009): What Makes a System Complex? - An Approach to Self-Organization and Emergence, in: AzizAlaoui, M.; Bertelle, C. (Eds.): From System Complexity to Emergent Properties, pp. 49-99, Springer, Berlin.

[6] Maurer, M.; Maisenbacher, S. (2013): Modeling and Analyzing Systems in Application, in: ICoRD'13, Global Product Development, pp. 705-717, Springer India, New Delhi.

[7] Schuh, G.; Lenders, M.; Kupke, D.; Nußbaum, C. (2008): Design for Changeability, in: ElMaraghy, $H$. (Ed.): Changeable and Reconfigurable Manufacturing Systems, pp. 251-266, Springer, London.

[8] Abdelkafi, N. (2008): Variety-Induced Complexity in Mass Customization, Erich Schmidt, Berlin.

[9] Schleich, H.; Schaffer, J.; Scavarda, L. F. (2007): Managing complexity in automotive production, 19th International Conference on Production Research, Valparaiso, Chile.

[10] Lindemann, U.; Maurer, M.; Braun, T. (2009): Structural Complexity Management. An Approach for the field of product development, Springer, Berlin.

[11] Hu, S. J; Zhu, X.; Wang, H.; Koren, Y. (2008): Product variety and manufacturing complexity in assembly systems and supply chains, in: CIRP Annals - Manufacturing Technology, Vol. 57, Issue 1, pp. 45-48.

[12] Heylighen F. (1999): The Growth of Structural and Functional Complexity during Evolution, in: Heylighen, F.; Bollen, J.; Riegler, A. (Eds.): The Evolution of Complexity, pp. 17-44, Kluwer Academic, Dordrecht.

[13] Feliz-Teixeira, J. M.; Carvalho Brito, A. E. S. (2009): Holistic Metrics, a Trial on Interpreting Complex Systems, in: Bertelle, C.; Duchamp, G. H. E.; Kadri-Dahmani, H. (Eds.): Complex Systems and Self-organization Modelling, pp. 21-28, Springer, Berlin.

[14] Marti, M. (2007): Complexity Management: Optimizing Product Architecture of Industrial Products, Dissertation, Universität St. Gallen, Deutscher Universitäts-Verlag, Wiesbaden.

[15] Samy, S. N.; EIMaraghy, H. (2012): Parts and Assembly Equipment Complexity Dependency Matrix, in: EIMaraghy, $\mathrm{H}$. (Ed.): Enabling Manufacturing Competitiveness and Economic Sustainability, pp. 452-457, Springer, Berlin.

[16] Landry, M.; Pascot, D.; Briolat, D. (1985): Can DSS evolve without changing our view of the concept of 'problem'?, in: Decision Support Systems, Vol. 1, Issue 1, January 1985, pp. 25-36.

[17] Fox, J. (1980): Making decisions under the influence of memory, Psychological Review, Vol. 87, No. 2, pp. 190- 211. 